\title{
The association between SPINK I and clinical outcomes in patients with prostate cancer: a systematic review and meta-analysis
}

This article was published in the following Dove Press journal:

OncoTargets and Therapy

22 June 2017

Number of times this article has been viewed

\author{
Xingming Zhang ${ }^{1, *}$ \\ Xiaoxue Yin ${ }^{2, *}$ \\ Pengfei Shen ${ }^{1, *}$ \\ Guangxi Sun' \\ Yaojing Yang' \\ Jiandong Liu' \\ $\mathrm{Ni} \mathrm{Chen}^{2}$ \\ Hao Zeng'
}

'Department of Urology, Institute of Urology, ${ }^{2}$ Department of Pathology,

West China Hospital, Sichuan

University, Chengdu, People's

Republic of China

*These authors contributed equally to this work
Correspondence: Ni Chen; Hao Zeng Guoxue Xiang 37\#, Chengdu 61004I, People's Republic of China Tel +86 I89 80602129 Fax +86288542245 I Email cdhx510@foxmail.com; kucaizeng@163.com
Abstract: Evidence of the prognostic role of serine peptidase inhibitor Kazal type 1 (SPINK1) in prostate cancer $(\mathrm{PCa})$ is controversial. The aim of this study was, therefore, to evaluate the association between SPINK1 and clinical outcomes in PCa. Searches were made of PubMed, Medline, Embase, and the China Biology Medicine disc (CBMdisc) up to January 2017. The Newcastle-Ottawa Scale was used to assess the risk of bias of included studies. RevMan software was used to perform meta-analysis, and the Grading of Recommendations Assessment, Development and Evaluation (GRADE) method was employed for assessing the quality of the evidence. Ten studies with 17,161 patients were included in the analysis. Random-effect models were adopted for all outcomes with significant heterogeneities. In patients treated with radical prostatectomy, SPINK1 was associated with biochemical recurrence (BCR) (hazard ratio $[\mathrm{HR}]=1.41,95 \%$ confidence interval $[\mathrm{CI}]: 1.01-1.97 ; P=0.04)$, but not PCa-specific mortality $(\mathrm{HR}=0.93,95 \% \mathrm{CI}: 0.33-2.57 ; P=0.88)$, and overall survival $(\mathrm{OS})(\mathrm{HR}=0.89$, 95\% CI: $0.58-1.35 ; P=0.57)$. In metastatic $\mathrm{PCa}$, SPINK1 was significantly associated with castration-resistant PCa-free survival (HR $=3.87,95 \% \mathrm{CI}: 1.87-8.00 ; P=0.0003$ ) and $\mathrm{OS}$ (HR $=2.59,95 \% \mathrm{CI}: 1.16-5.78 ; P=0.02$ ). However, the quality of the evidence was very low for all study outcome measures. In conclusion, although SPINK1 was not a predictor of PCa mortality or OS among patients who underwent radical prostatectomy, it may have prognostic value in metastatic PCa.

Keywords: SPINK1, clinical outcomes, prostate cancer, meta-analysis, systematic review

\section{Introduction}

Prostate cancer $(\mathrm{PCa})$ is the most common as well as the third leading cause of cancer-related deaths among men in Western countries. ${ }^{1}$ In previous decades, significant breakthroughs have been made in the diagnosis, treatment, and understanding of the genesis of PCa. However, advances in several areas, such as validated biomarkers in facilitating prostate-specific antigen screening, management of metastatic disease, and prognostic biomarkers to assist clinicians in predicting outcomes and decision making, still need to be made. ${ }^{2}$ So far, numerous biomarkers, including the expression of the E 26 (ETS) family of fusion genes, and serine peptidase inhibitor Kazal type 1 (SPINK1) overexpression, have been discovered in PCa.

In 1983, Huhtala et al first identified SPINK1 in urine samples of patients with gynecological cancers, which suggested that it may have significance as a tumor marker. ${ }^{3}$ Subsequently, SPINK1 was found to be abnormally expressed in solid tumors, including PCa. ${ }^{4}$ As a result of this, further studies were devoted to investigating whether this peptide was associated with the prognosis of PCa. Thus, Tomlins et al 
performed an analysis of molecular subtypes of PCa using SPINK1 and ETS-related gene (ERG) to predict clinical outcomes, and demonstrated the significant prognostic role of SPINK1 in PCa subtypes ${ }^{5}$ however, conflicting findings have been reported. Some studies found significant associations between SPINK1 and clinical outcomes while others demonstrated insignificant or even contrary correlations..$^{5-9}$

Therefore, we have conducted a systematic review and meta-analysis to evaluate the prognostic value of SPINK1 expression in patients with PCa. We have also assessed the current best evidence using the Grading of Recommendations Assessment, Development and Evaluation (GRADE) approach.

\section{Materials and methods}

Two researchers (XMZ and XXY) independently performed the searches, study selection, quality assessment, and data extraction of included studies. Disagreements were resolved by discussion or with the help of a third investigator (HZ).

\section{Eligibility and exclusion criteria for study selection}

The eligibility criteria included: 1) patients with pathologically confirmed PCa, 2) cohort or cross-sectional studies investigating the associations between SPINK1 and PCa clinical outcomes, and 3) no language restrictions for published studies. Exclusion criteria included reviews, reports that only focused on laboratory findings of expression profiles of SPINK1 without clinical prognostic outcome measures, and studies with only published abstracts.

Outcome measures included: 1) biochemical recurrence (BCR), 2) PCa-specific mortality (PCSM), 3) overall survival (OS), and 4) castration-resistant PCa (CRPC)-free survival. The definition of CRPC is provided in our previous study. ${ }^{10}$

\section{Data resources and searches}

Searches were made using PubMed (1950-2017.1), Medline (1966-2017.1), Embase (1947-2017.1), and the China Biology Medicine disc (CBMdisc, 1978-2017.1). The following terms and keywords were used: "trypsin inhibitor, Kazal pancreatic", "SPINK1", "pancreatic secretory trypsin inhibitor", "PSTI", "tumor-associated trypsin inhibitor", "TATI", and "prostate cancer". References in selected studies were searched for more studies, and experts in the field were consulted.

\section{Study selection and data extraction}

Two investigators independently reviewed the titles and abstracts of the search results. The full text versions of studies, which were potentially eligible, were then assessed. Participant characteristics, experimental techniques, outcome measures, results, and other pertinent data of each included study were extracted and recorded on data extraction forms, which were designed according to the guidelines specified in the Cochrane Handbook. ${ }^{11}$ We contacted authors to seek additional information where data were either not reported or not clear.

\section{Assessment of risk of bias}

Two reviewers (XMZ and XXY) independently assessed the risk of bias of included studies using the Newcastle-Ottawa Scale (NOS), which covers three main areas: selection, comparability, and outcome. ${ }^{12}$ Each study was categorized as low risk of bias ( $>7$ score), moderate risk of bias (5-7 score), or high risk of bias $(<5$ score $)$.

\section{Data synthesis}

RevMan software (version 5.3) was used to perform metaanalysis. Hazard ratios (HRs) and the corresponding 95\% confidence intervals (CIs) were synthesized with fixed or random effects models depending on the degree of heterogeneity. A random effect model was used when significant heterogeneity was considered at $P<0.10$ or $I^{2}>50 \%$.

Subgroup analysis was considered for the different groups of patients in the studies. Publication bias was assessed by creating a funnel plot using RevMan software. We applied GRADE approach to assess the quality of evidence recommended by the Cochrane Handbook. This approach specifies four levels of quality, ranging from high to moderate, low, and very low. ${ }^{11}$

\section{Results}

\section{Study characteristics}

Three hundred twenty-one studies were identified through electronic searches. One hundred one were excluded after duplicate publications. Full-texts of 38 studies were reviewed, and 13 published articles with 17,161 participants were finally included in the study (Figure 1). ${ }^{5-8,13-21}$ Characteristics of the included studies are summarized in Table 1. All studies were retrospective cohorts published between 2008 and 2016, of which three were conducted in the USA, ${ }^{5,13,17,21}$ two in Germany, ${ }^{14,15}$ two in China, ${ }^{16,18}$ and one each in France, ${ }^{6}$ Ireland, ${ }^{7}$ Finland, ${ }^{8}$ Korea, ${ }^{19}$ and Canada. ${ }^{20}$ Sample sizes ranged from 155 to 8,260. One study included patients with radical prostatectomy (RP) and/or transurethral resection of the prostate, ${ }^{13}$ and two studies included metastatic PCa (mPCa). ${ }^{8,18}$ Overall, seven studies with nine cohorts investigated the associations between 


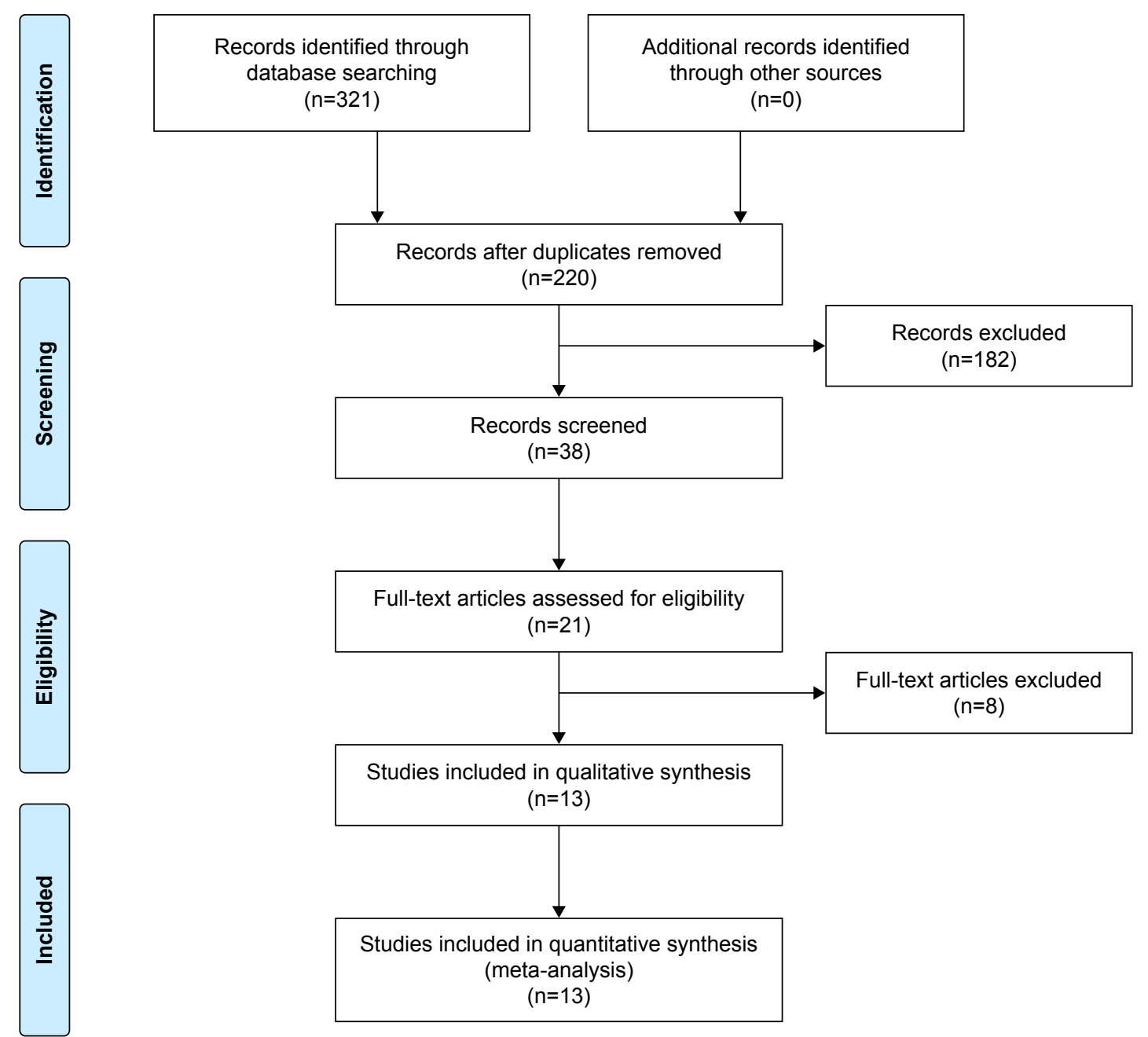

Figure I Preferred Reporting Items for Systematic Reviews and Meta-Analyses (PRISMA) flow diagram of search results.

SPINK1 expression and clinical outcomes in patients with RP. ${ }^{5-7,14-17}$

\section{Assessment of risk of bias for included studies}

According to the NOS, all included studies were of high quality with scores ranging from 7 to 9 (average 7.9). The most common source of bias was from outcome measures, for which most studies were unclear about the length or adequacy of follow-up (Table 2).

\section{Associations between SPINKI and clinical outcomes of PCa treated with RP}

As shown in Figure 2A, a pooled analysis indicated that expression of SPINK1 was associated with poor BCR-free survival in patients with PCa, with a HR of 1.41 (95\% CI: $1.01-1.97, P=0.04)$. No statistically significant associations were observed between SPINK1 expression and PCSMfree survival $(\mathrm{HR}=0.93,95 \% \mathrm{CI}$ : $0.33-2.57, P=0.88$;
Figure 2B) and $\mathrm{OS}(\mathrm{HR}=1.18,95 \% \mathrm{CI}: 0.55-2.52, P=0.67$; Figure 2C).

\section{Associations between SPINKI and clinical outcomes of $\mathrm{mPCa}$}

For patients with metastatic disease, SPINK1 expression was significantly associated with CRPC-free survival (HR $=3.87,95 \%$ CI: $1.87-8.00, P=0.0003$; Figure 2D), indicating that CRPC-free survival was shorter in patients with SPINK1-positive than SPINK1-negative tumors. Subgroup analysis suggested that SPINK1 expression was associated with shorter PCSM in patients with $\mathrm{mPCa}$ (HR $=2.6,95 \% \mathrm{CI}$ : 1.04-6.5; $P=0.04)$. However, only one study reported this outcome in $\mathrm{mPCa}$ patients. ${ }^{21}$ Because only one study investigated OS among $\mathrm{mPCa}$ patients, data synthesis could not be conducted. ${ }^{18}$ However, this study demonstrated that SPINK1 expression was negatively associated with OS ( $\mathrm{HR}=2.59,95 \% \mathrm{CI}$ : $1.16-5.78 ; P=0.02)$. 
Table I Characteristics of included studies

\begin{tabular}{|c|c|c|c|c|c|c|c|}
\hline Studies & Patients & $\mathbf{N}$ & Country & Age (years) & PSA (ng/mL) & Gleason score & Follow-up (months) \\
\hline Tomlins et $\mathrm{al}^{5}$ & RP/TURP & 971 & USA & NA & NA & NA & NA \\
\hline \multirow[t]{3}{*}{ Leinonen et $\mathrm{al}^{8}$} & Endocrine & 186 & Finland & $+: 71.6 \pm 8.4$ & $+: 63 \pm 104$ & $<7: 25.8 \%$ & $70(2-118)$ \\
\hline & Treated & & & $-: 73.8 \pm 6.8$ & $-: 105 \pm 429$ & $7: 29.0 \%$ & \\
\hline & & & & & & $>7: 41.9 \%$ & \\
\hline \multirow[t]{4}{*}{ Lippolis et al ${ }^{14}$} & RP & 3,385 & Germany & $\leq 49: 2.9 \%$ & $>4: 15.3 \%$ & $\leq 3+3: 38.8 \%$ & $65.8(I-219)$ \\
\hline & & & & $50-60: 26.0 \%$ & 4-10: $55.8 \%$ & $3+4: 46.4 \%$ & \\
\hline & & & & 60-70: 63.8\% & 10-20: $21.1 \%$ & $4+3: 11.6 \%$ & \\
\hline & & & & $>70: 7.3 \%$ & $>20: 7.8 \%$ & $\geq 4+4: 3.2 \%$ & \\
\hline \multirow[t]{4}{*}{ Grupp et al ${ }^{15}$} & RP & 8,260 & Germany & $<50: 2.9 \%$ & $<4: 12.6 \%$ & $\leq 3+3: 25.6 \%$ & $36.8(\mathrm{I}-228)$ \\
\hline & & & & $50-60: 24.8 \%$ & $4-10: 60.4 \%$ & $3+4: 14.0 \%$ & \\
\hline & & & & 60-70: $58.7 \%$ & 10-20: $19.4 \%$ & $4+3: 55.4 \%$ & \\
\hline & & & & $>70: 12.9 \%$ & $>20: 6.5 \%$ & $\geq 4+4: 4.3 \%$ & \\
\hline \multirow[t]{3}{*}{ Wang et $\mathrm{al}^{16}$} & RP & 211 & China & $\leq 70: 70.6 \%$ & $<4: 10.4 \%$ & $<7: 21.8 \%$ & NA \\
\hline & & & & $>70: 29.4 \%$ & $4-10: 12.8 \%$ & 7: $33.2 \%$ & \\
\hline & & & & & $>10: 76.8 \%$ & $>7: 45.0 \%$ & \\
\hline \multirow[t]{4}{*}{ Flavin et $\mathrm{al}^{7}$} & $\mathrm{RP}$ & 854 & Ireland & $65.4 \pm 6.0$ & $7.0 \pm 5.6$ & $2-6: 21.0 \%$ & $13.5 \pm 4.6$ \\
\hline & & & & & & $3+4: 37.0 \%$ & \\
\hline & & & & & & $4+3: 24.3 \%$ & \\
\hline & & & & & & $8-10: 17.6 \%$ & \\
\hline \multirow[t]{4}{*}{ Brooks et al ${ }^{17}$} & RP & 963 & USA & $61.7 \pm 7.2$ & $8.7 \pm 8.8$ & $<7: 94.0 \%$ & NA \\
\hline & & & & & & $3+4: 40.2 \%$ & \\
\hline & & & & & & $4+3: 12.5 \%$ & \\
\hline & & & & & & $>7: 10.1 \%$ & \\
\hline \multirow[t]{4}{*}{ Terry et a ${ }^{6}$} & RP & 279 & France & $64.1(44.2-75.2)$ & $13.5(1.3-119)$ & $<7: 43.0 \%$ & $50.1(I-190.5)$ \\
\hline & & & & & & $3+4: 13.7 \%$ & \\
\hline & & & & & & $4+3: 18.8 \%$ & \\
\hline & & & & & & $>7: 24.6 \%$ & \\
\hline \multirow[t]{5}{*}{ Tomlins et al ${ }^{13}$} & RP & I,577 & USA & $63(37.3-83)$ & $<10: 47.2 \%$ & $\leq 6: 16.2 \%$ & NA \\
\hline & & & & & 10-20: $19.7 \%$ & $7: 56.3 \%$ & \\
\hline & & & & & $>20: 15.6 \%$ & $8: 12.1 \%$ & \\
\hline & & & & & NA: $20.3 \%$ & $\geq 9: 17.9 \%$ & \\
\hline & & & & & & NA: $0.3 \%$ & \\
\hline \multirow[t]{4}{*}{ Pan et al ${ }^{18}$} & $\mathrm{mPCa}$ & 155 & China & $72.6 \pm 7.9$ & $70.2 \pm 35.4$ & $<7: 5.8 \%$ & $38.0(6-63)$ \\
\hline & & & & & & $3+4: 8.4 \%$ & \\
\hline & & & & & & $4+3: 26.5 \%$ & \\
\hline & & & & & & $>7: 59.4 \%$ & \\
\hline \multirow[t]{3}{*}{ Noh et al ${ }^{19}$} & RP & 64 & Korea & $+: 66.9 \pm 5.5$ & $+: 8.8 \pm 6.9$ & $<7: 27.9 \%$ & $49.2(11-111)$ \\
\hline & & & & $-: 63.9 \pm 5.3$ & $-: 13.2 \pm 10.0$ & 7: $52.9 \%$ & \\
\hline & & & & & & $>7: 19.1 \%$ & \\
\hline \multirow[t]{3}{*}{ Huang et $\mathrm{a}^{20}$} & $\mathrm{RP}$ & 178 & Canada & NA & NA & $<7: 31.1 \%$ & $42.0(0-142)$ \\
\hline & & & & & & $7: 56.1 \%$ & \\
\hline & & & & & & $>7: 12.7 \%$ & \\
\hline \multirow[t]{4}{*}{ Johnson et $\mathrm{al}^{21}$} & $\mathrm{mPCa}$ & 60 & USA & $+: 60$ (46-7I) & $<10: 56.7 \%$ & $\leq 6: 37.4 \%$ & $48.0(24-84)$ \\
\hline & & & & -: 59 (40-7I) & I0-20: $30.2 \%$ & $7: 46.2 \%$ & \\
\hline & & & & & $>20: 12.1 \%$ & 8: $12.9 \%$ & \\
\hline & & & & & & $\geq 9: 4.4 \%$ & \\
\hline
\end{tabular}

Notes: For age and PSA, data were represented as mean \pm SD, mean (range), or percentage. Follow-up was presented as median (range) or mean \pm SD.

Abbreviations: PSA, prostate specific antigen; mPCa, metastatic prostate cancer; NA, not available; RP, radical prostatectomy; SD, standard deviation; TURP, transurethral resection of prostate; +, SPINKI positive; -, SPINKI negative.

\section{Publication bias}

A funnel plot was used to assess the risk of publication bias. As shown in Figure 3, clear asymmetry was uncovered in the plot, which meant we could not exclude publication bias. Potential reasons for funnel plot asymmetry might be variations in study size and delayed publication, among other factors. However, because of the limited studies included in the analysis, it was difficult to obtain verification for this.

\section{Quality of evidence by GRADE approach}

As shown in Table 3, the quality of the evidence for BCRfree, PCSM-free, CRPC-free, and OS was graded as very 
Table 2 Risk of bias for included studies

\begin{tabular}{|c|c|c|c|c|c|c|c|c|c|c|}
\hline \multirow[t]{2}{*}{ Studies } & \multirow[t]{2}{*}{ Year } & \multicolumn{4}{|c|}{ Selection } & \multirow[t]{2}{*}{ Comparability } & \multicolumn{3}{|c|}{ Outcome } & \multirow[t]{2}{*}{ Total } \\
\hline & & (1) & (2) & (3) & (4) & & (5) & (6) & (7) & \\
\hline Tomlins et $\mathrm{al}^{5}$ & 2008 & $\star$ & $\star$ & $\star$ & $\star$ & $\star \star$ & $\star$ & $\star$ & is & 8 \\
\hline Leinonen et $\mathrm{al}^{8}$ & 2010 & $\star$ & $\star$ & $\star$ & $\star$ & $\star \star$ & $\star$ & $\Delta$ & th & 7 \\
\hline Lippolis et $\mathrm{al}^{14}$ & 2013 & $\star$ & $\star$ & $\star$ & $\star$ & $\star \star$ & $\star$ & $\star$ & th & 8 \\
\hline Grupp et $a^{15}$ & 2013 & $\star$ & $\star$ & $\star$ & $\star$ & $\star \star$ & $\star$ & $\star$ & it & 8 \\
\hline Wang et al ${ }^{16}$ & 2014 & $\star$ & $\star$ & $\star$ & $\star$ & $\star \star$ & $\star$ & is & its & 7 \\
\hline Flavin et $\mathrm{al}^{7}$ & 2014 & $\star$ & $\star$ & $\star$ & $\star$ & $\star \star$ & $\star$ & $\star$ & $\star$ & 9 \\
\hline Brooks et al ${ }^{17}$ & 2015 & $\star$ & $\star$ & $\star$ & $\star$ & $\star \star$ & $\star$ & is & its & 7 \\
\hline Terry et $\mathrm{al}^{6}$ & 2015 & $\star$ & $\star$ & $\star$ & $\star$ & $\star \star$ & $\star$ & $\star$ & $\vec{t}$ & 8 \\
\hline Tomlins et $\mathrm{al}^{13}$ & 2015 & $\star$ & $\star$ & $\star$ & $\star$ & $\star \star$ & $\star$ & $\star$ & $\overrightarrow{i t}$ & 8 \\
\hline Pan et $\mathrm{al}^{18}$ & 2016 & $\star$ & $\star$ & $\star$ & $\star$ & $\star \star$ & $\star$ & $\star$ & $\star$ & 9 \\
\hline Noh et al $^{19}$ & 2016 & $\star$ & $\star$ & $\star$ & $\star$ & $\star \star$ & $\star$ & $\star$ & $\star$ & 8 \\
\hline Huang et $\mathrm{al}^{20}$ & 2016 & $\star$ & $\star$ & $\star$ & $\star$ & $\star \star$ & $\star$ & $\star$ & its & 8 \\
\hline Johnson et $\mathrm{al}^{21}$ & 2016 & $\star$ & $\star$ & $\star$ & $\star$ & $\star \star$ & $\star$ & $\star$ & $\star$ & 8 \\
\hline
\end{tabular}

Notes: (1) Representativeness of the exposed cohort; (2) Selection of the nonexposed cohort; (3) Ascertainment of exposure; (4) Outcome not present at start; (5) Assessment of outcome; (6) Adequate follow-up length; (7) Adequacy of follow-up. The solid star represented adequate illustration of the corresponding items. The hollow star represented the opposite.

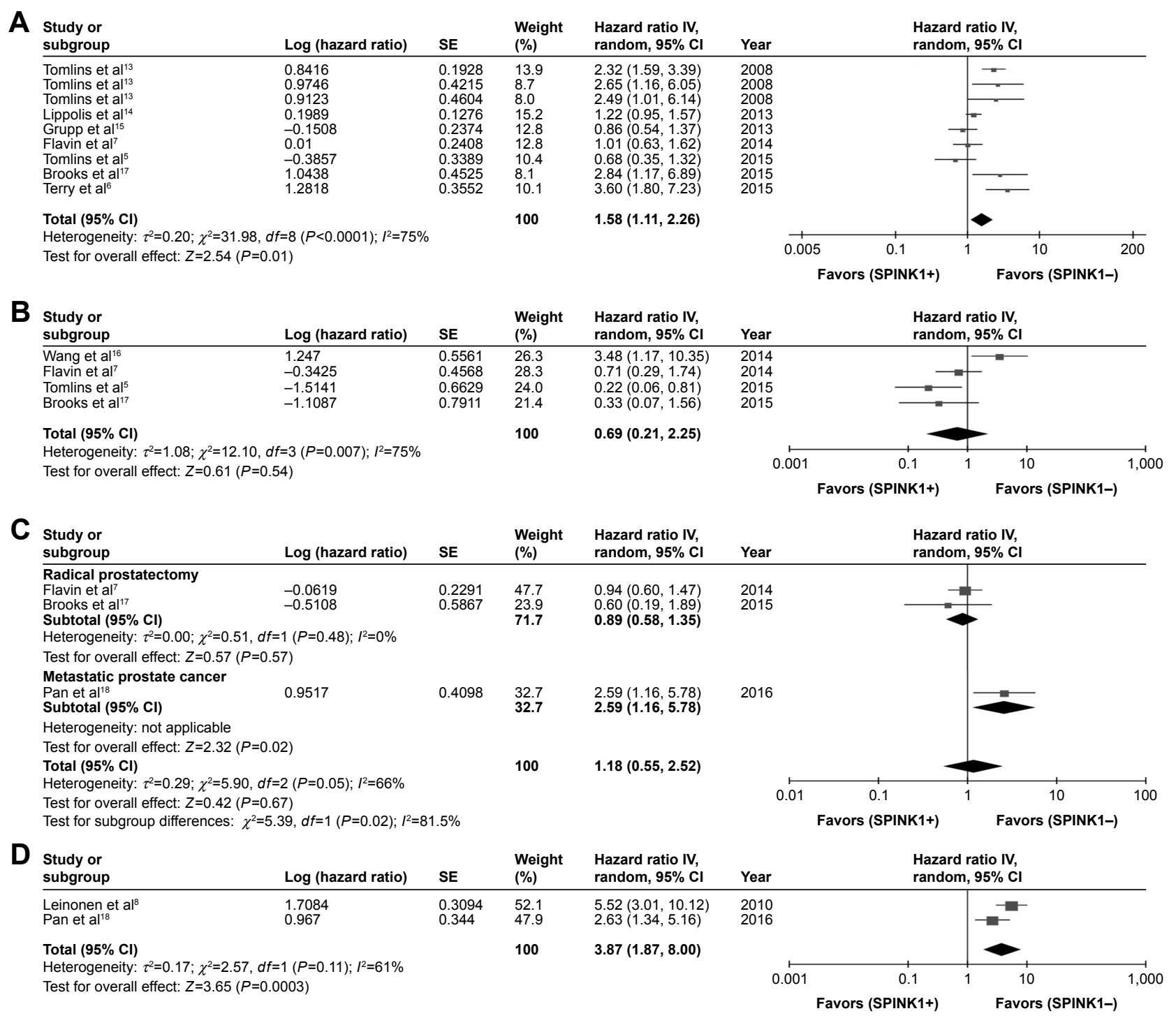

Figure 2 Forest plots of SPINKI expression and survival outcomes.

Notes: (A) Biochemical recurrence-free survival, (B) prostate cancer-specific mortality-free survival, (C) overall survival, and (D) CRPC-free survival.

Abbreviations: $\mathrm{Cl}$, confidence interval; CRPC, castration-resistant PC, ; df, degrees of freedom; SE, standard error; SPINKI, serine peptidase inhibitor Kazal type I. 


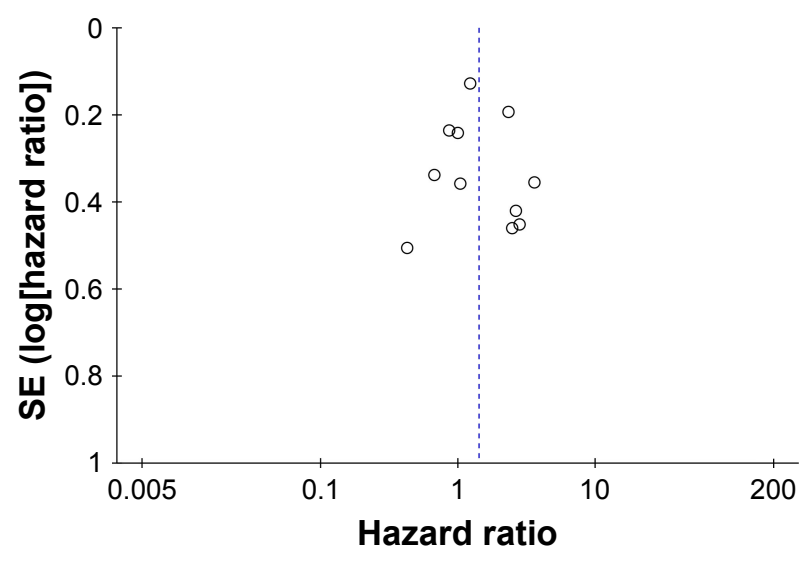

Figure 3 Funnel plot created by outcome of biochemical recurrence-free survival. Abbreviation: SE, standard error.

low in all instances. Serious inconsistencies were observed for BCR-free survival measures. Serious risk of bias, inconsistency, and imprecision were observed for PCSM- and CRPC-free survival outcomes as well. Finally, for OS, serious imprecision was found in both RP-treated and metastatic patients, and serious risk of bias was found in patients with metastatic disease.

\section{Discussion}

This is the first systematic review and meta-analysis to evaluate the association between SPINK1 and prognosis of patients with PCa. In general, we included 13 cohort studies involving 17,161 patients. There were nine studies with 11 cohorts that reported BCR-free survival among men treated with RP. For PCSM-free survival, four studies were included in the final pooled analysis for RP patients and one for $\mathrm{mPCa}$. However, only two studies reported CRPC-free survival and three studies reported OS. Eventually, we found that among patients treated with RP, SPINK1 was associated with BCRfree survival, but not PCSM-free survival or OS. In patients with metastatic disease, the associations were statistically significant between SPINK1- and CRPC-free survival and OS; however, both outcomes only included one study each. Although overall and cancer-specific survival were hard to evaluate, we still found a trend for an unfavorable role of SPINK1 in disease progression.

We noticed that the significant associations between clinical outcomes and SPINK1 expression were more prominent among patients with $\mathrm{mPCa}$. The distinction between metastatic and nonmetastatic disease implied that SPINK1 might have different roles at different stages of PCa progression. In 2015, Huang et al found that SPINK1 was upregulated at primary sites of patients with nodal $\mathrm{mPCa} \cdot{ }^{20}$ Furthermore, knockdown of SPINK1 in vitro could inhibit cell invasion but not proliferation. This finding might explain why SPINK1 was associated with adverse prognosis in aggressive prostate tumors, as opposed to less or no association with clinical outcomes in patients with less aggressive tumors. However, these findings are not conclusive because of the limited number of studies and small sample sizes reported in patients with metastatic disease. Further explorations of SPINK1 in advanced PCa are needed to verify these conclusions.

Although more evident in metastatic diseases, SPINK1 may play a role in patients treated with RP. The insufficient value of SPINK1 as an independent prognostic factor suggests there is an essential need to incorporate it with other biomarkers. In 2015, Tomlins et al combined SPINK1 and ETS gene fusions (most commonly involving ERG) to stratify patients into different molecular subtypes based on risks of clinical outcomes..$^{5}$ Although the best evidence indicated that ERG alone had no association with BCR- or PCSM-free survival, the combination of ERG and SPINK1 was associated with Gleason scores. ${ }^{22,23}$

The results of all studies included in this analysis were inconsistent. Therefore this meta-analysis was initiated

Table 3 Summary of findings of graded quality of evidence for all outcomes

\begin{tabular}{|c|c|c|c|c|c|c|c|c|}
\hline \multicolumn{5}{|c|}{ Quality assessment } & \multirow{2}{*}{$\begin{array}{l}\text { No of participants } \\
\text { (studies) }\end{array}$} & \multirow{2}{*}{$\begin{array}{l}\text { Relative effect } \\
(95 \% \mathrm{CI})\end{array}$} & \multirow[t]{2}{*}{ Quality } & \multirow[t]{2}{*}{ Importance } \\
\hline Risk of bias & Inconsistency & Indirectness & Imprecision & Other & & & & \\
\hline \multicolumn{9}{|c|}{ Biochemical recurrence-free survival } \\
\hline Not serious & Serious & Not serious & Not serious & None & 16,760 (9 studies) & HR I.4I (I.0I-I.97) & Very low & Critical \\
\hline \multicolumn{9}{|c|}{ PCSM-free survival for radical prostatectomy } \\
\hline Serious & Serious & Not serious & Serious & None & 3,630 (4 studies) & HR $0.69(0.2 I-2.25)$ & Very low & Critical \\
\hline \multicolumn{9}{|c|}{ CRPC-free survival } \\
\hline Serious & Serious & Not serious & Serious & None & 34 I (2 studies) & HR 3.07 (0.98-9.63) & Very low & Critical \\
\hline \multicolumn{9}{|c|}{ Overall survival for radical prostatectomy } \\
\hline Not serious & Not serious & Not serious & Serious & None & I,972 (2 studies) & HR $0.89(0.58-1.35)$ & Very low & Critical \\
\hline \multicolumn{9}{|c|}{ Overall survival for metastatic prostate cancer } \\
\hline Serious & Not serious & Not serious & Serious & None & I55 (I study) & HR 2.59 (I.16-5.78) & Very low & Critical \\
\hline
\end{tabular}

Abbreviations: $\mathrm{Cl}$, confidence interval; CRPC, castration-resistant PCa; HR, hazard ratio; PCSM, prostate cancer-specific mortality. 
to systematically assess these studies in the hope of understanding the real evidence for the prognostic role of SPINK1 in PCa. However, the differences among the included studies were too great to be conclusive. It is possible the disparities among these studies were as a result of issues with experimental, clinical, and/or statistical designs. The techniques and conditions for detecting the expression of SPINK1 in each study may also have been different. In addition, ethnicity, regional differences, and baseline characteristics of patients in the included studies might have contributed to the inconsistent results. Meanwhile, there were several limitations in our study. First, in the meta-analyses of all outcomes (BCR-, PCSM-, CRPC-free, and OS), significant heterogeneities were observed (Figure 2). Heterogeneities could be derived from different sample sizes or follow-up lengths among studies. Next, the quality of the evidence evaluated by the GRADE approach was very low, resulting in conservative recommendations. Moreover, because of the inherent characteristic of the slow progressive nature of most $\mathrm{PCa}$, it is difficult to obtain overall or PCa-specific survival in many studies. The analyses of CRPC-free survival and OS were restricted by the limited number of studies. More studies are needed to further investigate these outcomes. In addition, we were unable to extract eligible data for final analyses even in some studies that reported similar outcomes.

Most of the patients were treated with RP, and only a few had metastatic disease. It has been proven that biomarkers expressed at different disease sites or periods of disease progression might not reflect the current immune-active microenvironment. For instance, programmed death ligand-1 (PD-L1), a novel biomarker in PCa, is discordantly expressed in metastatic sites and primary tumors. ${ }^{24,25}$ Therefore, we believe it is necessary to further evaluate the association between SPINK1 expression and clinical outcomes in metastatic patients, especially the expression of SPINK1 in the metastatic site or stage.

\section{Conclusion}

This is the first systematic review and meta-analysis to evaluate the association between SPINK1 and clinical outcomes among patients with $\mathrm{PCa}$. We found that patients with SPINK1-positive PCa had shorter BCR-free survival (RP group), CRPC-free survival (mPCa), and OS (mPCa) than SPINK1-negative patients. SPINK1 was not a predictor of PCa mortality or OS among patients treated with RP. However, very low quality of evidence assessed by the GRADE approach suggests that high-quality data are necessary to further verify these conclusions.

\section{Acknowledgments}

This work was supported by the Natural Science Foundation of China (NSFC 81402110, 81672547, 81272820, and 81172439 ) and the Science and Technology Support Program of Sichuan Province (2015SZ0230-3).

\section{Author contributions}

$\mathrm{NC}$ and $\mathrm{HZ}$ conceived the study. XMZ and XXY carried out the search process and screened for included studies. XMZ, XXY, GXS, and YJY extracted the data and assessed risk of bias for included studies. XMZ, PFS, and JDL conducted the analysis. XMZ and XXY were involved in writing the paper. All authors contributed toward data analysis, drafting and revising the paper and agree to be accountable for all aspects of the work.

\section{Disclosure}

The authors report no conflicts of interest in this work.

\section{References}

1. Siegel RL, Miller KD, Jemal A. Cancer statistics, 2016. CA Cancer JClin. 2016;66(1):7-30.

2. Attard G, Parker C, Eeles RA, et al. Prostate cancer. Lancet. 2016; 387(10013):70-82.

3. Huhtala ML, Kahanpää K, Seppälä M, Halila H, Stenman UH. Excretion of a tumor-associated trypsin inhibitor (TATI) in urine of patients with gynecological malignancy. Int J Cancer. 1983;31(6):711-714.

4. Rasanen K, Itkonen O, Koistinen H, Stenman UH. Emerging roles of SPINK1 in cancer. Clin Chem. 2016;62(3):449-457.

5. Tomlins SA, Alshalalfa M, Davicioni E, et al. Characterization of 1577 primary prostate cancers reveals novel biological and clinicopathologic insights into molecular subtypes. Eur Urol. 2015;68(4):555-567.

6. Terry S, Nicolaiew N, Basset V, et al. Clinical value of ERG, TFF3, and SPINK1 for molecular subtyping of prostate cancer. Cancer. 2015; 121(9):1422-1430.

7. Flavin R, Pettersson A, Hendrickson WK, et al. SPINK1 protein expression and prostate cancer progression. Clin Cancer Res. 2014;20(18): 4904-4911.

8. Leinonen KA, Tolonen TT, Bracken H, et al. Association of SPINK1 expression and TMPRSS2:ERG fusion with prognosis in endocrinetreated prostate cancer. Clin Cancer Res. 2010;16(10):2845-2851.

9. Smith SC, Tomlins SA. Prostate cancer SubtyPINg biomarKers and outcome: is clarity emERGing? Clin Cancer Res. 2016;20(18): 4733-4736.

10. Zhao T, Liao B, Yao J, et al. Is there any prognostic impact of intraductal carcinoma of prostate in initial diagnosed aggressively metastatic prostate cancer? Prostate. 2015;75(3):225-232.

11. Schünemann HJ, Oxman AD, Vist GE, et al. Chapter 12: Interpreting results and drawing conclusions. In: Higgins JPT, Green S, editors. Cochrane Handbook for Systematic Reviews of Interventions. Version 5.1.0 [updated March 2011]. The Cochrane Collaboration, $2011 ; 12.1-12.20$.

12. Wells G, Shea B, O'Connell D, et al. The Newcastle-Ottawa Scale (NOS) for assessing the quality of nonrandomised studies in meta-analyses. Available from: www.ohri.ca/programs/clinical_epidemiology/oxford. asp. Accessed March 15, 2016.

13. Tomlins SA, Rhodes DR, Yu J, et al. The role of SPINK1 in ETS rearrangement-negative prostate cancers. Cancer Cell. 2008;13(6): $519-528$. 
14. Lippolis G, Edsjo A, Stenman UH, Bjartell A. A high-density tissue microarray from patients with clinically localized prostate cancer reveals ERG and TATI exclusivity in tumor cells. Prostate Cancer Prostatic Dis. 2013;16(2):145-150.

15. Grupp K, Diebel F, Sirma H, et al. SPINK1 expression is tightly linked to 6q15- and 5q21-deleted ERG-fusion negative prostate cancers but unrelated to PSA recurrence. Prostate. 2013;73(15):1690-1698.

16. Wang C, Wang L, Su B, et al. Serine protease inhibitor Kazal type 1 promotes epithelial-mesenchymal transition through EGFR signaling pathway in prostate cancer. Prostate. 2014;74(7):689-701.

17. Brooks JD, Wei W, Hawley S, et al. Evaluation of ERG and SPINK1 by immunohistochemical staining and clinicopathological outcomes in a multi-institutional radical prostatectomy cohort of 1067 patients. PLoS One. 2015;10(7): 0132343.

18. Pan X, Zhang X, Gong J, et al. The expression profile and prognostic value of SPINK1 in initially diagnosed bone metastatic prostate cancer. Prostate. 2016;76(9):823-833.

19. Noh BJ, Sung JY, Kim YW, Chang SG, Park YK. Prognostic value of ERG, PTEN, CRISP3 and SPINK1 in predicting biochemical recurrence in prostate cancer. Oncol Lett. 2016;11(6):3621-3630.

20. Huang KC, Evans A, Donnelly B, Bismar TA. SPINK1 overexpression in localized prostate cancer: a rare event inversely associated with ERG expression and exclusive of homozygous PTEN deletion. Pathol Oncol Res. Epub 2016 Oct 13.
21. Johnson MH, Ross AE, Alshalalfa M, et al. SPINK1 defines a molecular subtype of prostate cancer in men with more rapid progression in an at risk, natural history radical prostatectomy cohort. J Urol. 2016; 196(5):1436-1444.

22. Pettersson A, Graff RE, Bauer SR, et al. The TMPRSS2:ERG rearrangement, ERG expression, and prostate cancer outcomes: a cohort study and meta-analysis. Cancer Epidemiol Biomarkers Prev. 2012;21(9): 1497-1509.

23. Huang KC, Begin LR, Palanisamy N, Donnelly B, Bismar TA. SPINK1 expression in relation to PTEN and ERG in matched primary and lymph node metastatic prostate cancer: implications for biomarker development. Urol Oncol. 2015;34(5):235.e1-e10.

24. Mukherji D, Jabbour MN, Saroufim M, et al. Programmed death-ligand 1 expression in muscle-invasive bladder cancer cystectomy specimens and lymph node metastasis: a reliable treatment selection biomarker? Clin Genitourin Cancer. 2016;14(2):183-187.

25. Giraldo NA, Becht E, Pagès F, et al. Orchestration and prognostic significance of immune checkpoints in the microenvironment of primary and metastatic renal cell cancer. Clin Cancer Res. 2015;21(13): $3031-3040$
OncoTargets and Therapy

\section{Publish your work in this journal}

OncoTargets and Therapy is an international, peer-reviewed, open access journal focusing on the pathological basis of all cancers, potential targets for therapy and treatment protocols employed to improve the management of cancer patients. The journal also focuses on the impact of management programs and new therapeutic agents and protocols on

\section{Dovepress}

patient perspectives such as quality of life, adherence and satisfaction The manuscript management system is completely online and includes a very quick and fair peer-review system, which is all easy to use. Visit http://www.dovepress.com/testimonials.php to read real quotes from published authors. 